\title{
Dynamic Coalition Formation and the Core*
}

\author{
Tone Dieckmann \\ Department of Economics, National University of Ireland, Maynooth, Ireland \\ Tone.Dieckmann@may.ie \\ Ulrich Schwalbe \\ Department of Economics, University of Hohenheim, 70593 Stuttgart, Germany \\ schwalbe@uni-hohenheim.de \\ June 1998, revised: January 2000
}

This paper presents a dynamic model of endogenous coalition formation in cooperative games with transferable utility. The players are boundedly rational. At each time step, a player decides which of the existing coalitions to join, and demands a payoff. These decisions are determined by a best-reply rule, given the coalition structure and allocation in the previous period. Further, the players experiment with myopically suboptimal strategies whenever there are potential gains from trade. We establish an isomorphism between the set of absorbing states of the process and the set of core allocations, and show that the process converges to one of these states with probability one whenever the core is non-empty. These results do not require superadditivity of the characteristic function, and they carry over to the case of coalitional values depending on the coalition structure.

Keywords: TU Games, Coalition Formation, Bounded Rationality, Core

JEL classification: $\mathrm{C} 71 ; \mathrm{C} 73$

\section{Introduction}

Most equilibrium concepts in games, both cooperative and non-cooperative, are static by definition. For example, the core of a cooperative game is the set of feasible allocations that cannot be blocked by any coalition of players. This implies that core allocations are stable in the sense that, once a core allocation is achieved, no subset of players can gain by deviating from it. However, the theory fails to explain how the players arrive at a core allocation, or at equilibrium in general. Reaching a certain allocation (core or otherwise) requires the completion of two a priory unrelated processes on the part of the players: Coalition formation and bargaining about how to split the surplus within each of the coalitions. Once coalitions have formed, the surplus, or payoff accruing to each coalition, is determined by the characteristic function of the game. However, the concept of the core relies on allocations, i. e. individual payoffs, rather than coalitional surplus. The link between the two concepts,

${ }^{*}$ We wish to thank Andreas Blume for his suggestions and comments, and we are grateful to the participants of the workshop Coalition Formation and Applications to Economics, Bilbao, April 1999, for helpful comments and discussions. 
the characteristic function on the one hand and the core on the other, is an implicit bargaining process that detemines the division of the coalitional payoff between the members of the coalition. The theory of cooperative games ignores both the issue of coalition formation and the bargaining process.

The present paper addresses these issues. We are interested in questions like: How do coalitions form, and how do the players decide on the division of the coalitional payoff? How do coalition structures change over time? Which of the possible coalition structures will the players eventually arrive at, and what will be the resulting allocation?

Dynamic learning models provide a framework for analysing these questions. These models are based on the assumption that players are only boundedly rational, and follow simple adaptation rules which are based on myopic optimization. While dynamic learning models have been widely applied to non-cooperative games (Ellison (1993), Kandori, Mailath, and Rob (1993), Young (1993) are among the most seminal works), relatively little research in this field has been done with respect to cooperative games.

This paper provides a dynamic model of endogenous coalition formation. The setup is similar to models of dynamic learning in non-cooperative games with local interaction and player mobility (e. g. Dieckmann 1999). In these models, players can move freely between several locations at each time step, and interaction, i. e. the play of a game, takes place only between players inhabiting the same location. A strategy for each player thus consists of a location choice and an action for the game. Similarly, in the context considered here, a player's strategy consists of a coalition choice and a demand for his share of the coalitional payoff. That is, at each time step, a player decides which of the existing coalitions to join, and demands a share of the payoff, which is determined by the characteristic function. A player will join (or quit) a coalition if and only if he believes it is in his own best interest to do so. Therefore, these decisions are determined by a (non-cooperative) best-reply rule: A player switches coalitions only if his expected payoff in the new coalition exceeds his current payoff, and he demands the most he can get conditional on feasibility. More precisely, the player observes the prevailing coalition structure and the demands of the other players. Expectations are adaptive in the sense that each player expects the present coalition structure and demand to prevail in the next period. The player then chooses the coalition in which he can demand the highest possible payoff, given the demands of the other members of that coalition, and subject to feasibility. As time goes to infinity, the process generated by all players' adopting the best-reply rule converges to an absorbing state (or set of states). Under the pure best-reply process, absorbing states do not necessarily involve core allocations. However, if we allow the players to experiment, i. e. deviate from the best-reply rule with a small probability whenever there exists a potentially better outcome, all absorbing states will be identified with core allocations.

Despite the fact that there have been several experimental studies on coalition formation (e.g. Sauermann (1978), Rapoport, Kahan, Funk, Horowitz (1979)), there are 
only very few theoretical papers dealing with the problem of coalition formation in a dynamic context. These are the works by Shenoy $(1979,1980)$, Packel (1981), and, most closely related to this paper, Agastya (1997, 1999). Agastya (1997) presents a dynamic model of social learning where, in each period, each player observes a random sample of demand vectors drawn from a finite history, and adjusts his demand according to a best-reply rule. This rule differs from the one used in the present paper in that players maximize their expected payoffs, conditional on the probability that their demand is compatible with a feasible allocation. Agastya assumes that, whenever there exists any coalition structure for which a player's demand is feasible, given the other players' demands, the player receives the payoff he demands with probability one. The process of coalition formation is not modeled. Agastya derives an isomorphism between the set of absorbing states of the learning process and the core of the game. Agastya (1999) extends the model by introducing "mistakes" on the part of the players, and finds that the set of stochastically stable states is a subset of the set of core states.

Our model departs from Agastya in several respects. First, Agastya entirely abstracts from coalition formation, and focuses on allocations. The bargaining process considered by Agastya is simple: Each player announces his demand, i. e. the payoff he asprires to get. If there exists a coalition structure such that the vector of all players' demands is feasible, then each player will get his demand with probability one. Agastya (1999) writes: "it is reasonalbe to assume that eventually, a maximal coalition (in terms of set inclusion) whose demands are feasible forms." ${ }^{1}$ Indeed, for the class of superadditive games considered by Agastya, this assumption is reasonable. Our model, however, is not restricted to superadditive games. We allow for the case that, e. g. large organizations may operate less efficiently than the sum of their constituent parts. In this case, it is not reasonable to assume that a maximal coalition will form. Instead, we model the coalition formation process explicitly, by the players' choosing both a demand and a coalition in each period. The coalition structure in each period is thus endogenously determined, which allows us to study how coalitions of players evolve over time. Further, letting the players choose their coalitions makes our model applicable to a wider range of economic problems, such as local public good economies, or $\mathrm{clubs}^{2}$, where individuals care not only about allocations but also about the number and/or the characteristics of people in their coalition.

A further advantage of our approach is that it allows us to extend the model to the case of coalition structure dependent payoffs, which we do in section 6 . In many economic situations, a coalition's payoff is not independent of other coalitions, e. g. duopoly, or public good games where coalitions can free ride on other coalitions' contributions to the provision of a public good.

A model of endogenous coalition formation in a dynamic context is provided by

\footnotetext{
${ }^{1}$ Agastya (1999), p. 215.

${ }^{2}$ For a survey of the literature, see Wooders (1999); for an application of adaptive learning to local public good economies see Dieckmann (1999).
} 
Packel (1981). He defines a Markov process on the set of outcomes, i. e. payoff allocations. Given the individual preferences over all outcomes, the transition probability from one state to another is proportional to the number of minimal coalitions that prefer the new state to the old one. The core is then defined by the union of the absorbing states of the process. The stochastic solution of the process is the probability distribution obtained by letting time go to infinity. It follows that, whenever the core in non-empty, the stochastic solution places probability one on the set of core allocations. Moreover, Packel shows that, if the strong core (i. e. the singleton set of undominated outcomes that can be reached from every other outcome with positive probability) is non-empty, the stochastic solution places probability one on that state. The main difference between Packel's model and our own is that Packel abstracts from behaviour rules on the individual level, while we explicitly model the players' coalition choice and demands with respect to payoff.

For the sake of completeness, we would like to mention other strands of the literature concerned with bargaining and coalition formation, which are (albeit somewhat remotely) related to our model. These approaches come under the heading of "endogenous coalition formation", e. g. Raj and Vohra (1996, 1997), or "non-cooperative models of coalition formation", e. g. Perry and Reny (1994). This literature provides models of bargaining that lead to some kind of stable coalition structure. However, these models are entirely different in spirit since they focus on a one-shot game, i. e. the process ends once a stable coalition structure has emerged. In contrast, our model is inherently dynamic: Coalition choice and bargaining are carried out repeatedly according to a predetermined adaptation process, the best-reply rule, which never ends. To illustrate this difference by an example, consider the model of Perry and Reny (1994). Building on a model by Kalai, Postlewaite and Roberts (1978), Perry and Reny use a continous time model where at each point in time a player can make a proposal consisting of a coalition to which the player would like to belong and a payoff allocation for the members of that coalition. If the proposal is accepted by the members of the coalition, these players drop out of the game and the remaining players continue bargaining. In effect, Perry and Reny associate with every cooperative game with transferable utility a noncooperative sequential game, and show that the stationary subgame perfect equilibria of this game conincide with the core allocations of the cooperative game, thus providing a non-cooperative motivation for core allocations. However, this approach is static rather than dynamic: One notion of equilibrium, the core, is justified by another, the Nash equilibrium. The model does not provide an explanation of how such an equilibrium is reached, or if it is reached at all. While models of non-cooperative foundations of the core are interesting in their own right, they are conceptually different from our dynamic approach.

The remainder of the paper is organized as follows. The next section introduces the basic model. We provide a definition of the core for non-superadditive games, and describe the adaptation process. Section 3 shows the existence of absorbing states. In section 4, we modify the process by introducing noise. Section 5 provides 
our main result, namely that the modified process converges to a state involving a core allocation with probability one as time tends towards infinity, regardless of the initial state in which the process starts. Section 6 extends the model to the case of coalition structure dependent payoffs, and section 7 concludes.

\section{The Model}

Let $N=\{1, \ldots, n\}$ denote the set of players. Any subset $S \subset N$ is called a coalition. The set of all nonempty coalitions $2^{N} \backslash\{\emptyset\}$ is denoted by $\mathcal{N}$. A game in characteristic function form with transferable utility (or for short a TU-game) is defined by a mapping $v: \mathcal{N} \rightarrow \mathbf{R}$, the characteristic function. This function $v$ associates with any nonempty coalition the maximal total payoff for that coalition. Note that the payoff for a coalition does not depend on the behaviour of other coalitions. A vector of payoffs $x=\left(x_{1}, \ldots, x_{n}\right)$, one for each player, is called an allocation. An allocation is feasible for coalition $S$ if $\sum_{i \in S} x_{i} \leq v(S)$.

Usually, cooperative game theory makes several assumptions on the characteristic function, e. g. superadditivity or convexity of $v$. A characteristic function is superadditive if for any two disjoint coalitions $S$ and $T$ the following relation holds: $v(S)+v(T) \leq v(S \cup T)$, i. e. if the members of $S$ and $T$ unite, they can do at least as well as by remaining separate. Convexity, an even stronger assumption, is defined as follows: A characteristic function is convex if for any two coalitions $S$ and $T$ : $v(S)+v(T) \leq v(S \cup T)+v(S \cap T)$.

If the cooperative game is superadditive, an allocation is called feasible if $\sum_{i \in N} x_{i}$ $\leq v(N)$, and it is efficient if equality holds. Assume that an allocation $x$ has been proposed. If a group of players can form a coalition which can secure its members a higher payoff than the proposed allocation, this coalition will block the proposal. Formally, the allocation $x$ will be blocked by a coalition $S$ if $\sum_{i \in S} x_{i}<v(S)$. This idea is employed by the solution concept of the core of a game, which is defined by the set of all feasible allocations that cannot be blocked by any coalition. That is, an allocation $x$ is in the core if

$$
\sum_{i \in N} x_{i}=v(N) \quad \text { and } \quad \sum_{i \in S} x_{i} \geq v(S) \quad \forall S \subseteq N
$$

However, superadditivity or convexity of the characteristic function are quite restrictive requirements. As an example, consider the following simple production economy. Suppose that each player is endowed with capital $e_{i}$, and endowments are transformed into a single good by a real valued production function $f$. If we define the characteristic function by $v(S)=f\left(\sum_{i \in S} e_{i}\right)$, superadditivity of the characteristic function implies non-decreasing returns to scale.

In order to be able to deal with non-superadditive characteristic functions, we redefine the concepts of feasibility and the core in the following way. Let $\mathcal{C}$ denote the set of all possible coalition structures, i. e. the set of all partitions of $N$, where a typical coalition structure is denoted by $C$. We say that an allocation is feasible 
if the total payoff to the players does not exceed the highest possible outcome, i. e. the outcome that can be achieved under the most favourable coalition structure: $x$ is feasible if $\sum_{i \in N} x_{i} \leq \max _{C \in \mathcal{C}} \sum_{S \in C} v(S)$.

Definition 1 For any TU-game $v$, an allocation $x \in \mathbf{R}^{n}$ is a core allocation if

(i) $\sum_{i \in N} x_{i}=\max _{C \in \mathcal{C}} \sum_{S \in C} v(S)$, and

(ii) $\sum_{i \in S} x_{i} \geq v(S) \quad \forall S \subset N$.

In words: The allocation $x$ is feasible and efficient, and there are no blocking coalitions.

Note that, if $v$ is superaditive, $\max _{C \in \mathcal{C}} \sum_{S \in C} v(S)$ is equal to $v(N)$, such that condition $(i)$ of the definition reduces to the familiar expression $\sum_{i \in N} x_{i}=v(N)$.

In order to exclude trivial games, we assume that no single player can do better on his own than as a member of any coalition.

Assumption 1 For all coalitions $S: \sum_{i \in S} v(\{i\}) \leq v(S)$.

We now turn to the best-reply process by which coalitions evolve. Each player $i$ 's strategic variables are his coalition choice and his demand $d_{i}$, i. e. the share of the surplus generated by $v$ he aspires to get.

Time is discrete. In each period $t$, all players announce their respective demands, and the allocation is determined in a way to be described shortly. Realized payoffs depend on the compatibility of the individual demands in each coalition. The allocation in period $t+1$ is determined as follows. If the demands within a coalition $S$ are feasible, each member of $S$ will receive his demand. Otherwise, each $i \in S$ gets his reservation payoff $v(\{i\})$. That is, for each $i \in S,^{3}$

$$
x_{i}=\left\{\begin{array}{cl}
d_{i} & \text { if } \sum_{i \in S} d_{i} \leq v(S) \\
v(\{i\}) & \text { otherwise. }
\end{array}\right.
$$

Thus, a player's reservation payoff can be interpreted as a disagreement outcome: If negotiations fail in the sense that the members of a coalition are unable to agree upon the division of the surplus, each has to fall back upon his own resources. Assumption 1 ensures that the allocation $x_{i}=v(\{i\})$ for all $i \in S$ is feasible.

We say that the demand vector $d=\left(d_{i}\right)_{i \in N}$ is feasible given coalition structure $C$ if $\sum_{i \in S} d_{i} \leq v(S)$ for all $S \in C$.

A players opportunity to revise his strategy arises at random. In each period $t$, each player independently takes a random draw from a Bernoulli trial. With probability $\gamma \in(0,1)$, the draw produces the outcome "adjust". ${ }^{4}$ If this happens, the player adjusts his current strategy as follows. He observes the prevailing coalition structure

\footnotetext{
${ }^{3}$ In what follows, the superscript $t$ is omitted in order to simplify notation.

${ }^{4}$ Nöldeke and Samuelson (1993) refer to this event as "receiving the learn draw".
} 
$C^{t} \in \mathcal{C}$, as well as the demand vector $d^{t}=\left(d_{1}^{t}, \ldots, d_{n}^{t}\right)$. On the basis of this information, the player decides whether to join any of the existing coalitions $S \in C^{t}$ or to form a singleton coalition, and simultaneaously announces his demand $d_{i}^{t+1}$. This determines the coalition structure and the demand for the next period. The state of the game at each point in time is therefore characterized by the the tuple $\omega^{t}=\left(C^{t}, d^{t}\right)$.

For reasons of mathematical tractability, we restrict the players' demands to be chosen from a finite set. Suppose there is a smallest accounting unit $\delta$ with $0<\delta<1$, and $\delta$ sufficiently small.

Assumption 2 Each player's demand is restricted to the finite set $D_{i}$ of all integral multiples of $\delta$ in the closed intervall $\left[v(i), \max _{C \in \mathcal{C}} \sum_{S \in C} v(S)\right]$.

Given any coalition structure $C$, the strategies available to player $i$ are

$$
\Sigma_{i}(C):=\left\{\left(S_{i}, d_{i}\right) \mid S_{i}=S \cup\{i\} \text { for some } S \in C \cup\{\emptyset\}, d_{i} \in D_{i}\right\} .
$$

Player $i$ 's strategy set is then

$$
\Sigma_{i}=\cup_{C \in \mathcal{C}} \Sigma_{i}(C) .
$$

As the number of possible coalitions and coalition structures as well as the possible demands are all finite, we are dealing with a finite strategy space. Thus, the notions of feasibility and efficiency, and therefore the definition of a core alloction, have to be modified.

Definition 2 For any TU-game $v$, an allocation $x \in \mathbf{R}^{n}$ is a core allocation if

(i) $x_{i} \in D_{i} \forall i \in N, \sum_{i \in N} x_{i} \leq \max _{C \in \mathcal{C}} \sum_{S \in C} v(S)$, and if the inequality is strict, then $\sum_{i \in N} x_{i}+\delta>\max _{C \in \mathcal{C}} \sum_{S \in C} v(S)$,

(ii) $\sum_{i \in S} x_{i} \geq v(S) \quad \forall S \subset N$.

In what follows, the term "core allocation" will refer to definition 2.

The players are myopic. A player who is selected to move seeks to maximize his expected payoff for the next period, conditional on feasibility. Further, the players have adaptive expectations. Each player, when it is his turn to adjust his strategy, expects the current coalition structure and the demands of the other players to prevail in the next period. Note that, if $\gamma$, the probability of getting the opportunity to adjust, is small, these expectations are "almost" rational: If $\gamma$ is small, the probability that two players revise their strategies at a time is close to zero.

The players' choice of coalition membership is restricted by the current coalition structure. Given any coalition structure $C$, the player faces three options: $(i)$ he can stay in his present coalition, $(i i)$ he can join any of the other coalitions $S \in C$, or $(i i i)$ he can form the singleton coalition $\{i\}$ (if this is different from $(i)$ ). At this point, coordination problems may arise due to incompatibility of players' plans. For 
instance, suppose in some period $t$, player $i$ is forming the singleton coalition $\{i\}$, but plans to join some coalition $S$ in $t+1$, while another player, $j$, plans to join $\{i\}$ in $t+1$. Now, if both $i$ and $j$ get the chance to adjust their strategies at the same time, their plans will be incompatible. To solve this problem, we assume that each player, when he gets the chance to move, can always leave his current coalition and join the coalition of his choice, but no player can be forced to stay in any coalition. That is, player $i$ will join $S$, and $j$ will end up in the singleton coalition $\{j\}$. If coalitions are interpreted as firms, the assumption implies that employees can quit (and join another firm) without notice. An alternative interpretation would be to imagine that coalitions form at certain "meeting points", i. e. different locations, or clubs, where all players assembled at the same location form a coalition. A player who wants to join a coalition has to go to that coalition's meeting point. In the above example, this means that $i$ would go to the meeting point of coalition $S$, and at the same time $j$ would go to the location formerly occupied by $i$, which he will find deserted. This assumption ensures that the coalition structure in each period is always well defined.

As a myopic maximizer, the player chooses that coalition which promises him the highest feasible payoff. His demand is then determined by his maximum expected payoff given the other players' demands, i. e.

$$
d_{i}(\omega)=\max _{S \in C \cup\{\emptyset\}} v(S \cup\{i\})-\sum_{\substack{j \neq i \\ j \in S}} d_{j} \text { s.t. } d_{i} \in D_{i},
$$

and the chosen coalition $S_{i}(\omega)$ is one in which $d_{i}$ can be achieved, given the demands of its members, i. e.

$$
S_{i}(\omega) \in\left\{\arg \max _{S \in C \cup\{\emptyset\}} v(S \cup\{i\})-\sum_{\substack{j \neq i \\ j \in S}} d_{j}\right\} .
$$

If this coalition is not unique, the player randomizes, choosing each of the maximizing coalitions with positive probability. However, we assume that a player switches coalitions only if his maximum expected payoff from doing so strictly exceeds his current payoff, i. e. if $d_{i}(\omega)>x_{i}$.

Given the finite strategy space, the individual adaptation rules define a finite Markov chain with state space $\Omega=\left\{\omega=(C, d) \mid C \in \mathcal{C}, d \in \times_{i \in N} D_{i}\right\}$. Let $S(i)$ denote the coalition player $i$ belongs to in any state $\omega$. The transition probability from state $\omega$ to $\omega^{\prime}$ with demand $d_{i}^{\prime}$ and coalition $S^{\prime}(i)$ is then

$$
p_{\omega \omega^{\prime}}=\Pi_{i \in N} \gamma \beta_{i}\left(\omega^{\prime} \mid \omega\right)
$$

where $\beta_{i}$ is defined by the best-reply rule, i. e. $\beta_{i}\left(\omega^{\prime} \mid \omega\right)>0$ if and only if $d_{i}^{\prime}=d_{i}(\omega)$ as defined in (2), and $S^{\prime}(i)$ is a maximizer of (3). In what follows, we will refer to this Markov chain as the best-reply process. 
As coalition structures evolve over time, it is interesting to analyse under what conditions the process will settle down to a stable coalition structure, where no player has an incentive to switch anymore. To this purpose, we employ the concept of ergodic sets.

Definition $3 A$ set $\mathcal{E} \subset \Omega$ is ergodic if for any $\omega \in \mathcal{E}, \omega^{\prime} \notin \mathcal{E}, p_{\omega \omega^{\prime}}=0$, and no nonempty proper subset of $\mathcal{E}$ has this property. Singleton ergodic sets are called absorbing states, i. e. $\omega \in \Omega$ is absorbing if $p_{\omega \omega}=1$.

Ergodic sets are thus minimal sets with the property that, once the process has entered such a set, it will remain in that set forever after, and the process will perpetually vacillate between the states in the ergodic set. A stable coalition structure will evolve if the process reaches a singleton ergodic set, an absorbing state. In an absorbing state, no player has an incentive to revise his strategy, given the prevailing coalition structure. The following lemma characterizes absorbing states.

Lemma 1 A state $\omega=(C, d)$ is an absorbing state of the best-reply process if and only if the following three conditions are met:

(i) $d_{i}=x_{i} \geq v(\{i\}) \forall i \in N$,

(ii) $\forall S \in C: \sum_{i \in S} d_{i} \leq v(S)$, and if the inequality is strict, then

$$
\sum_{i \in S} d_{i}+\delta>v(S)
$$

(iii) $\forall i \in N: \nexists S \in C: v(S \cup\{i\})-\sum_{\substack{j \neq i \\ j \in S}} d_{j}>x_{i}$

Proof. Condition $(i)$ states that the allocation corresponding to the state equals the demand vector. This is the case if and only if demands are feasible, clearly a necessary condition for the state being absorbing. Further, each player must get at least his reservation payoff, or else he would deviate from the state by forming a singleton coalition.

Condition $(i i)$ states that the allocation within each coalition is feasible and efficient. If the sum of the demands fell short of $v(S)$, at least one member of the coalition could increase his demand without affecting feasibility, unless the difference between $v(S)$ and the total demand is smaller than the smallest accounting unit $\delta$.

Finally, (iii) ensures that no player has an incentive to switch coalitions. While each of these conditions is necessary for an absorbing state, the three conditions together are sufficient: If all three conditions are met, no player can possibly gain by deviating from that state.

Absorbing states thus represent individually stable equilibria in the definition of Greenberg (1994). 


\section{Absorbing States}

Absorbing states represent stable strategy configurations in the sense that no player wants to revise his strategy. Assumption 1 provides a sufficient condition for the existence of absorbing states.

Theorem 1 If assumption 1 holds, the best-reply process has at least one absorbing state.

Proof. Assumption 1 implies $v(N) \geq \sum_{i \in N} v(\{i\})$. An absorbing state is constructed as follows: Assume that the grand coalition $\{N\}$ has formed and each player receives a payoff $x_{i} \geq v(\{i\})$ with $\sum_{i \in N} x_{i}=v(N)$. As payoffs are individually rational, no player has an incentive to form the singleton coalition. Further, as all players are members of the grand coalition, there is no other coalition to join. Finally, as the demands sum up to $v(N)$, no player can increase his demand. Thus, under the condition of the theorem, each element of the set $\Omega^{N}(v):=\{\omega=(C, d) \mid C=$ $\left.\{N\}, d_{i} \geq v(\{i\}) \forall i \in N, \sum_{i \in N} d_{i}=v(N)\right\}$ is an absorbing state.

The set $\Omega^{N}$ is the set of states involving the grand coalition where all players' demands are indiviually rational, and the demand vector is feasible and efficient given $\{N\}$. That is, the set of allocations associated with $\Omega^{N}$ is the set of all efficient imputations achievable by the grand coalition. Stated otherwise, a sufficient condition for an absorbing state to exist is that the game is essential in the definition X.2.6 of Owen (1995).

It is well known from the theory of finite Markov chains that, as time tends towards infinity, the process converges to an ergodic set with probability one, irrespective of the initial state. ${ }^{5}$ However, the best-reply process considered here typically exhibits multiple absorbing states and/or ergodic sets, and which of these will eventually be reached depends on the initial state in which the process starts. As the title of this paper suggests, we are interested in deriving conditions under which the process converges towards states involving core allocations. We are now going to explore the relationship between absorbing states and the core of a game.

Clearly, if $x$ is a core allocation, every state $\omega=(C, d)$ with $d=x$ where $d$ is feasible given $C$ must be absorbing: Condition $(i)$ of definition 1 implies $(i)$ and $(i i)$ of lemma 1, and the non-existence of blocking coalitions (condition (ii) of definition 1) implies condition (iii) of lemma 1.

However, the converse is not true, as the following examples show.

Example 1: Let $N=\{1,2,3\}$, and $v(\{i\})=2$ for all $i \in N, v(S)=5$ for $|S|=2$, and $v(N)=8$. The state $\omega=(\{N\},(4,2,2))$ is absorbing, but the allocation $x=(4,2,2)$ is not in the core since it is blocked by the coalition $\{2,3\}$.

Example 2: The 3-player majority game. $N=\{1,2,3\}, v(\{i\})=0$ for all $i \in N$, and $v(S)=10$ for $|S| \geq 2$. The core is empty, but any state $\omega=(\{N\}, d)$ with $\sum_{i \in N} d_{i}=10$ is absorbing. In addition to absorbing states, this example exhibits

\footnotetext{
${ }^{5}$ E. g. Kemeny and Snell (1976), Theorem 3.1.1 on p. 43.
} 
non-singleton ergodic sets. This is the case if the process starts in a state where one of the players receives the entire surplus of 10, e. g.

$$
\begin{aligned}
\omega^{1} & =(\{\{1,2\},\{3\}\},(10,0,0)) \\
\omega^{2} & =(\{\{1\},\{2,3\}\},(10,10,0)) \\
\omega^{3} & =(\{\{1\},\{2,3\}\},(0,10,0)) \\
\omega^{4} & =(\{\{1,3\},\{2\}\},(0,10,10)) \\
\omega^{5} & =(\{\{1,3\},\{2\}\},(0,0,10)) \\
\omega^{6} & =(\{\{1,2\},\{3\}\},(10,0,10)) \\
\omega^{7} & =\omega^{1} .
\end{aligned}
$$

The transitions between the states are effected as follows. In $\omega^{1}$, only player 2 is not playing a best reply. Thus, if player 2 gets the chance to adjust, he will join player 3 to form the coalition $\{2,3\}$, and demand $d_{2}^{2}=10$. If any of the other two players get the chance to adjust, they will not change their strategies. Therefore, $\omega^{2}$ is constructed from $\omega^{1}$ by player 2's strategy adjustment. In $\omega^{2}$, player 1's demand is not feasible for the coalition $\{1\}$. Player 1's best reply is to adjust his demand to zero, and stay in the singleton coalition. Players 2 and 3 are playing best replies in $\omega^{2}$. The new state $\omega^{3}$ is thus obtained from $\omega^{2}$ by adjusting player 1's demand. In $\omega^{3}$, the only player who does not play a best reply is player 3 . If he gets the chance to adjust, he will join player one to form the coalition $\{1,3\}$, and demand $d_{3}^{4}=10$. This leads to $\omega^{5}$, and so forth.

Once this set is entered, the process will forever "cycle", i. e. visit the states $\omega^{1}$ to $\omega^{6}$ in a fixed order. However, due to the stochastic nature of the adjustment process, the states are not visited at regular intervals of time. For instance, switching from $\omega^{1}$ to $\omega^{2}$ requires that player 2 gets the opportunity to adjust his strategy, an event that occurs with probability $\gamma$. As a consequence, the process might remain in state $\omega^{1}$ for several periods, until player 2 is able to adjust, and the number of periods the process stays in each state is random.

Thus, using the pure best-reply rule described above, the process might get trapped in a dominated allocation, or in a "cycle". Similar phenomena have been observed in other contexts, e. g. in the theory of conventions where it could be shown (cf. Berninghaus and Schwalbe (1996), Young (1993)) that a population of players will adopt an inefficient convention, in problems concerning the choice of a technology as in Arthur (1989), or in the theory of the core of an exchange economy when trade is restricted to a bilateral exchange as in Feldman (1973). We will now slightly modify the players' adaptation rule by allowing them to experiment with (myopically) suboptimal strategies. 


\section{Best Reply with Experimentation}

The result that the set of absorbing states may comprise inefficient or non-equilibrium states is not new, as the literature on evolutionary models of non-cooperative games shows (e. g. Kandori et al. (1993), Young (1993)). A solution to this problem is to introduce noise to the dynamics by allowing the players to pick myopically suboptimal strategies with a small probability, which is supposed to model evoultionary mutations, or "trembles", that are interpreted as mistakes in the socio-economic context. The limit distribution of this stochastic process is then derived by letting the probability of mistakes go to zero, and the states that receive positive probability under the limit distribution are called stochastically stable. The set of stochastically stable states is a subset of the set of absorbing states of the model with no noise. Thus, stochastic stability can be regarded as a "refinement" of the concept of absorbing states, in the sense that some of these states are singled out.

While Agastya (1999) adopts this method to narrow down the set of absorbing states, we pursue a different approach. Instead of allowing for mistakes, we introduce experiments on the part of the players. To motivate the idea of experimenting, suppose the system has entered an absorbing state that is not in the core. That is, there exists a blocking coalition which cannot be realized due to the current coalition structure. For instance, in example 1, players 2 and 3 could secure themselves a payoff of 5 , but the coalition $\{2,3\}$ cannot be realized because, according to the rules of the game, the players can switch only between existing coalitions, or form a singleton coalition.

Now, if we assume that the characteristic function is common knowledge among the players, each player is aware of the fact that a blocking coalition exists. While players 2 and 3 would want to form this coalition, the absence of communication possibilities prevents them from doing so. The only way to enable the coalition $\{2,3\}$ to form would be for one of the two players to form a singleton coalition, say $\{2\}$, and player 3 to join in the next period. This, however, contradicts our assumption that players switch coalitions only if they expect a strictly higher payoff. We now modify the model by allowing the players to switch to myopically suboptimal strategies (with a small probability) whenever they are a member of a potentially blocking coalition that cannot be reached under the prevailing coalition structure.

Assumption 3 In any state $\omega=(C, d)$, whenever there exists a coalition $S^{\prime} \notin C$ with $v\left(S^{\prime}\right)>\sum_{i \in S^{\prime}} d_{i}$, each player $i \in S^{\prime}$ chooses a best reply with probability $1-\epsilon$, and takes each strategy $\left(S_{i}, d_{i}\right) \in \Sigma_{i}(C)$ with probability $\epsilon /\left|\Sigma_{i}(C)\right|$ when he gets the opportunity to revise his strategy. The probability $\epsilon$ is independent across players and states.

In words, a player chooses any combination of coalition and demand with positive but small probability. Experimenting enables a player to destabilize a state involving a dominated allocation, at the cost of a temporary loss in payoff. Experimenting differs from the concept of "trembles" or "mutations" mentioned above in that 
experimenting is done deliberately: the players experiment if and only if there exists an outcome that is potentially better for them, whereas mutations occur in any state with uniform probability. We believe it is reasonable to assume that players will be inclined to experiment only if there is a chance that the outcome of the experiment may eventually improve their situation.

Note that experimenting requires a slightly higher degree of rationality than the simple best-reply rule, since the players have to be able to look two periods ahead, not just one. However, they are still far from being fully rational, which would require choosing a strategy in each period that maximizes their expected payoff for all future periods, taking into account the entire history of the game. In contrast, experimenting requires to look forward only two periods, and to take only the current state into account.

We will refer to the best-reply process modified by assumption 3 as the best-reply process with experimentation. Clearly, states involving dominated demand vectors cannot be absorbing states of this process, since there is a positive probability that a player experiments. As a result, we get the following theorem.

Theorem 2 The set of demand vectors associated with an absorbing state of the best-reply process with experimentation coincides with the set of core allocations:

(i) If $x$ is a core allocation, then all $\omega=(C, d)$ with $d=x$ and $d$ being feasible given $C$ are absorbing states.

(ii) If $\omega=(C, d)$ is an absorbing state, then $d$ is a core allocation.

Proof. Part $(i)$ is obvious: If $x=d$ is in the core, no player can gain either by switching coalitions or by changing his demand. Moreover, as no blocking coalitions exist, no player experiments. Thus, the state is absorbing.

Part $(i i)$ : Suppose $\omega=(C, d)$ is absorbing, but $d$ is not in the core. If $d$ is not a core allocation, there exists a coalition $S$ with $v(S)>\sum_{i \in S} d_{i}$. Assumption 3 implies that all $i \in S$ experiment with probability $\epsilon$. Thus, there is a positive probability that the state is left, a contradiction to $\omega$ being absorbing.

It is clear that the set of absorbing states of the process with experimentation is a subset of the set of absorbing states of the pure best-reply process. The following example illustrates the theorem.

Example 3: Suppose the number of players $n$ is even, $v(\{i\})=0$, and $v(S)=|S|+2$ for all $S \subseteq N$ with $|S| \geq 2$. Here the optimal coalition size, i. e. the number of members that maximizes the (average) per capita payoff is $|S|=2$. The unique core allocation is $x_{i}=2$ for all $i \in N$.

Note that, if a player's current payoff is less than one, and there are at least two coalitions, his best reply will be to switch coalitions and demand a payoff of one. Thus, the set of absorbing states of the best-reply process is $\Omega^{N}(v) \cup\{\omega=(C, d)|| S \mid \geq$ $\left.2, \sum_{i \in S} d_{i}=v(S) \forall S \in C, d_{i} \geq 1 \forall i \in N\right\}$, where $\Omega^{N}(v)$ is defined as in the proof of theorem 1 . The set of absorbing states of the process with experimentation 
is the subset of states where players form pairwise coalitions and demand 2, i. e. $\left\{\omega=(C, d)|| S \mid=2 \forall S \in C, d_{i}=2 \forall i \in N\right\}$. The common demand vector of these states coincides with the core allocation of the game.

\section{Convergence to Absorbing States}

Theorem 2 states that, if the core is non-empty, each core allocation can be reached in an absorbing state, and any absorbing state can be associated with a core allocation. However, the theorem does not guarantee that a core allocation will actually be reached by the process. While the theory of Markov chains provides a result that ensures convergence towards an ergodic set, it does not guarantee that such a set be singleton, i. e. an absorbing state (see example 2). The following theorem excludes the possibility that the process gets "trapped" in an ergodic set other than an absorbing state.

Theorem 3 If the core is non-empty, the best-reply process with experimentation will converge to an absorbing state with probability one as time tends towards infinity.

Proof. We prove the result by showing that all ergodic sets are singletons. Suppose that there exists an ergodic set $\mathcal{E} \subset \Omega$ with $|\mathcal{E}| \geq 2$. We establish a contradiction by showing that $\mathcal{E}$ contains a state from which there is a path to an absorbing state. Theorem 2 ensures that none of the states in $\mathcal{E}$ involve a core allocation. (This follows from the fact that all core allocations are reached in an absorbing state, and ergodic sets are minimal, i. e. cannot contain other ergodic sets.) As a consequence, each state $\omega=(C, d) \in \mathcal{E}$ is characterized by the existence of a coalition $S \notin C$ with $v(S)>\sum_{i \in S} d_{i}$. That is, there are some players who will experiment with suboptimal strategies if they get the chance to adjust their strategies. There is a positive probability that all these players get the chance to adjust in some period $t$, and no other player gets this chance. Moreover, there is a positive probability that all players who experiment form the singleton coalition and demand their reservation payoff, i. e. they pick the strategy $(\{i\}, v(\{i\}))$. Thus, all states resulting from any $\omega \in \mathcal{E}$ by replacing $S(i)$ with $S \backslash\{i\}$ and adding $\{i\}$ in $C$ and replacing $d_{i}$ with $v(\{i\})$ for all players $i$ who experiment with the singleton coalition can be reached with positive probability from $\omega$. Denote the set of all such states by $R(\omega)$. It follows that $\cup_{\omega \in \mathcal{E}} R(\omega) \subset \mathcal{E}$, since all states in that set are reached with positive probability. By the same argument as above, elements of $R(\omega)$ cannot involve core allocations. Repeating the same procedure as before, replacing $\mathcal{E}$ by $R(\omega)$ for each $\omega \in \mathcal{E}$ in the argument, we get a set $R^{2}(\omega)$ for each $\omega \in \mathcal{E}$. Again, the union of all the $R^{2}(\omega)$ must be in $\mathcal{E}$.

It is clear that, after repeating this procedure a finite number $k$ of times, the set $\cup_{\omega \in \mathcal{E}} R^{k}(\omega)$ contains the state where either $(a)$ each player forms the singleton coalition, or $(b)$ some players form singleton coalitions, and those who are not in a 
singleton coalition have no incentive to experiment. That is, for players in nonsingleton coalitions, there is no potentially blocking coalition that would give one of them a higher payoff than the current $x_{i}$. However, it could be that these players' demands sum up to less than $v(S)$. In this case, there is a positive probability that some of these players get the opportunity to adjust their demands, and no other player gets this opportunity. The resulting state satisfies $v(S)-\delta<\sum_{i \in S} x_{i} \leq v(S)$ for all non-singleton coalitions $S$. Thus, $\mathcal{E}$ contains a state $\omega^{\prime}=\left(C^{\prime}, d^{\prime}\right)$ with the following property: Either $S^{\prime}(i)=\{i\}$ and $d_{i}=v(\{i\})$ for all $i \in N$, or, if there are coalitions $S^{\prime} \in C^{\prime}$ with $\left|S^{\prime}\right| \geq 2$, then there exists an absorbing state $\omega^{c}=\left(C^{c}, d^{c}\right)$ such that $S^{\prime}=S^{c}$ for some $S^{c} \in C^{c}$, and $d_{i}^{\prime}=d_{i}^{c}$ for all $i \in S^{\prime}$, for all $S^{\prime}$ with $\left|S^{\prime}\right| \geq 2$. (As the core is non-empty, an absorbing state exists. Further, as members of $S^{\prime}$ play best replies and do not experiment, their demands must be part of a core allocation.)

Starting from $\omega^{\prime}$, an absorbing state $\omega^{c}=\left(C^{c}, d^{c}\right)$ can be reached in one step. For each $i \in N$, we denote by $S^{c}(i)$ the coalition $i$ belongs to in the absorbing state. Suppose the process is in state $\omega^{t}=\omega^{\prime}$. We now construct a vector of moves on the part of the players that yields $\omega^{t+1}=\omega^{c}$. Assumption 1 ensures that, in state $\omega^{\prime}$, for each player who forms the singleton coalition, there exists a potentially better coalition, and thus each $i$ with $S(i)=\{i\}$ experiments with probability $\epsilon$. Further, all players who are in a non-singleton coalition in $\omega^{\prime}$ do not experiment. Now $\omega^{c}$ can be reached as follows. For each $T^{c} \in C^{c}$, fix one player $i$ with $S^{c}(i)=T^{c}$. Call this player $i\left(T^{c}\right)$. Now suppose all players who are in a singleton coalition get the opportunity to adjust their strategies, and that all these players experiment. This happens with probability $(\gamma \epsilon)^{m}$, where $m$ is the number of singleton coalitions in $\omega^{\prime}$. The experimenting players choose the following strategies. Each player $i$ experiments with $S_{i}=S^{c}(i)$ and $d_{i}=d_{i}^{c}$ : For every coalition $T^{c} \in C^{c}$, the player $i\left(T^{c}\right)$ stays in the singleton coalition $\left\{i\left(T^{c}\right)\right\}$ and demands $d_{i}^{c}$. All other players $j \in T^{c}$ join the coalition $\left\{i\left(T^{c}\right)\right\}$, and demand $d_{j}^{c}$. The resulting state is $\omega^{c}$. That probability $p_{\omega^{\prime} \omega^{c}}$ is equal to $\left(\gamma \epsilon /\left|\Sigma_{i}\left(C^{\prime}\right)\right|\right)^{m}$, where $\left|\Sigma_{i}\left(C^{\prime}\right)\right|=\left|C^{c}\right|\left|D_{i}\right|$ is the number of strategies available to player $i$ in state $\omega^{\prime}$ (there are $\left|C^{c}\right|$ coalitions to choose from). Thus, there is a positive probability that an absorbing state is reached when the system starts in state $\omega^{\prime}$. This is a contradiction to $\omega^{\prime}$ being an element of an ergodic set. It follows that all ergodic sets are singletons, which completes the proof.

Theorems 2 and 3 together ensure that, if the core of a game is non-empty, the bestreply process with experimentation will reach a core allocation with probability one as time goes to infinity, no matter where the process starts.

\section{Coalition Structure Dependent Payoffs}

The characteristic function form of a game is based on the assumption that the value of a coalition is independent of the coalition structure. In many economic 
situations, however, this is not the case. For instance, consider a Cournot-oligopoly model where the firms have the option to form binding coalitions, i. e. cartels. Then, the payoff to each coalition depends on the entire coalition structure in the industry. Our model can easily be extended to allow for this case.

The idea of coalitional payoffs depending on the underlying coalition structure is captured by the concept of a partition function introduced by Raj and Vohra (1996). A partition function $\nu: \mathcal{N} \times \mathcal{C} \rightarrow \mathbf{R}$ assigns to each coalition $S$ in coalition structure $C$ a value $\nu(S, C)$.

The concept of a blocking coalition has to be redefined. Given a coalition structure $C$ and allocation $x$, let $C_{S}$ be the coalition structure resulting from $C$ by removing all members of $S$ from $C$ and adding coalition $S$. For instance, if $C=$ $\{\{1,2,3\},\{4,5,6\}\}$ and $S=\{2,4\}$, then $C_{S}=\{\{1,3\},\{2,4\},\{5,6\}\}$.

Coalition $S$ can block $x$ if $\nu\left(S, C_{S}\right)>\sum_{i \in S} x_{i}$. That is, $S$ can block $x$ if its value under the new coalition structure, i. e. the one resulting from the deviation of $S$, exceeds their payoff under $x$. The blocking of an allocation thus depends on the coalition structure.

Definition 4 A state $(C, x)$ is stable if

(i) $\sum_{i \in N} x_{i}=\max _{C \in \mathcal{C}} \sum_{S \in C} \nu(S, C)$, and

(ii) $\sum_{i \in S} x_{i} \geq \nu\left(S, C_{S}\right) \quad \forall S \subset N$.

The first condition states that the allocation in a stable state is feasible and efficient. The second condition ensures that no blocking coalitions exist.

As the players' payoffs depend on the coalition structure, a player's reservation payoff is not uniquely defined, since the payoff a player can get as a singleton coalition $\{i\}$ depends on the prevailing partition of $N \backslash\{i\}$. We define player $i$ 's reservation payoff by the minimum payoff he can possibly get under any coalition structure.

Definition 5 Player $i$ 's reservation payoff under partition function $\nu$ is defined by

$$
r_{i}(\nu)=\min _{C \in \mathcal{C}, C \supset\{i\}} \nu(\{i\}, C) .
$$

Given this definition, assumption 1 can be rewritten as follows.

Assumption 4 For all coalition structures $C$ and all $S \in C: \sum_{i \in S} r_{i} \leq \nu(S, C)$.

In the special case of a coalition structure independent characteristic function, i. e. $\nu(S, C)=\nu\left(S, C^{\prime}\right)=: v(S)$ for all $C, C^{\prime}$ containing $S, r_{i}=v(\{i\})$, such that assumption 4 is equivalent to the original assumption 1.

Given any state $\omega=(C, d)$, the allocation in this state is determined by player $i$ in coalition $S$ receiving

$$
x_{i}= \begin{cases}d_{i} & \text { if } \sum_{i \in S} d_{i} \leq \nu(S, C) \\ r_{i} & \text { otherwise }\end{cases}
$$


The best-reply rule has to be slightly modified. When choosing a coalition, a player has to take into account the fact that coalitional payoffs vary with the coalition structure. Given any state $\omega=(C, d)$, a player, when it is his turn to move, picks that coalition for which the surplus $\nu(S \cup\{i\}, \cdot)-\sum_{j \in S} d_{j}$ is maximized for the coalition structure resulting from i's strategy adjustment. More precisely, his coalition choice is determined by

$$
S_{i}(\omega) \in\left\{\arg \max _{S \in C \cup\{\emptyset\}} \nu\left(S \cup\{i\}, C_{\{i\}}\right)-\sum_{j \in S} d_{j}\right\},
$$

where $C_{\{i\}}$ is the coalition structure resulting from $C$ by player $i$ 's strategy adjustment, keeping all other players' strategies fixed.

Similarly, a players demand is given by

$$
d_{i}(\omega)=\max _{S \in C \cup\{\emptyset\}} \nu\left(S \cup\{i\}, C_{\{i\}}\right)-\sum_{j \in S} d_{j} \text { s.t. } d_{i} \in D_{i} .
$$

It is immediately obvious that any stable state $(C, d)$ must be absorbing under the best-reply rule without experimentation. Assume that, in any state $(C, d)$, if there exists a coalition $S \in C_{S}$ with $\nu\left(S, C_{S}\right)>\sum_{i \in S} d_{i}$, all members of $S$ experiment, i. e. pick a best reply with probability $1-\epsilon$ and any other strategy with probability $\epsilon$. Arguments analogous to the proofs of theorems 2 and 3 can be used to establish an isomorphism between absorbing states and stable allocations, and ensure convergence to an absorbing state with probability one as time tends towards infinity.

\section{Conclusion}

This paper proposed a dynamic process of endogenous coalition formation in cooperative games. Coalition membership and the allocation of payoffs in each period are determined by a simple adaptation rule that is based on myopic best replies on the part of the players, and players experiment with suboptimal strategies whenever there is a chance that this might lead to a preferred coalition structure. Under a very mild condition concerning the characteristic function, absorbing states are shown to exist. Moreover, if the core is non-empty, the process will converge to a state involving a core allocation with probability one.

The idea to apply dynamic learning processes to cooperative games is quite novel. The new aspect introduced in the present paper is the explicit formulation of a bargaining process that simultaneously determines the coalition structure and the allocation in each time period. This formulation allows us to dispense with restrictive assumptions on the characteristic function such as superadditivity or convexity, which in turn ensures that our model is applicable to a large class of games. What is more, our results carry over to the case of coalition structure dependent payoffs. 


\section{References}

Agastya, M. (1997): 'Adaptive Play in Multiplayer Bargaining Situations', Review of Economic Studies 64, 411-426.

Agastya, M. (1999): 'Perturbed Adaptive Dynamics in Coalition Form Games', Journal of Economic Theory 89, 207-233.

Arthur, W.B. (1989): 'Competing Technologies, Increasing Returns, and Lock-In by Historical Events', The Economic Journal 99, 116-131.

Berninghaus, S., U. Schwalbe (1996): 'Conventions, Local Interaction and Automata Networks', Journal of Evolutionary Economics 6, 297-312.

Dieckmann, T. (1999): 'The Evolution of Conventions with Mobile Players', Journal of Economic Behavior and Organization 38, 93-111.

Dieckmann, T. (2000): 'A Dynamic Model of a Local Public Good Economy with Crowding', Working paper.

Ellison, G. (1993): 'Learning, Local Interaction, and Coordination', Econometrica 61, 1047-1071.

Feldman, A. (1973): 'Bilateral Trading Processes, Pairwise Optimality, and Pareto Optimality', Review of Economic Studies 40, 463-473.

Greenberg, J. (1994): Coalition Structures, Handbook of Game Theory, Elsevier Science B. V., ed. R. J. Aumann and S. Hart, Vol. 2, Chapter 37, 1305-1337.

Kalai, E., A. Postlewaite, J. Roberts (1979): 'A Group Incentive Compatible Mechanism Yielding Core Allocations', Journal of Economic Theory 20, 13-22.

Kandori, M., G. Mailath, and R. Rob (1993): 'Learning, Mutation, and Long Run Equilibria in Games', Econometrica 61, 29-56.

Kemeny, J.G., and J.L. Snell (1976): Finite Markov Chains. Springer-Verlag, New York.

Nöldeke, G., and L. Samuelson (1993): 'An Evolutionary Analysis of Backward and Forward Induction', Games and Economic Behavior 5, 425-454.

Owen, G. (1995): Game Theory, Academic Press.

Packel, E.W. (1981): 'A Stochastic Solution Concept for $n$-Person Games', Mathematics of Operations Research 6, 349-363.

Perry, M., P.J. Reny (1994): 'A noncooperative View of Coalition Formation and the Core', Econometrica $62,795-817$.

Raj, D., R. Vohra (1996): 'A Theory of Endogenous Coalition Structure', mimeo.

Raj, D., R. Vohra (1997): 'Equilibrium Binding Agreements', Journal of Economic Theory 73, 30-78.

Rapoport, A., J.P. Kahan, S.G. Funk, A.D. Horowitz (1979): Coalition Formation by Sophisticated Players, Springer.

Sauermann, H. (ed.) (1978): Coalition Forming Behavior, Mohr.

Shenoy, P.P. (1979): 'On Coalition Formation: A Game-Theoretical Approach', International Journal of Game Theory 8, 133-164.

Shenoy, P.P. (1980): 'A Dynamic Solution Concept for Abstact Games' Journal of Optimization Theory and Applications 32, 151-169.

Wooders, M.H. (1999): 'Multijurisdictional Economies, the Tiebout Hypothesis, and Sorting', Perspective, Proc. Natl. Acad. Sci. USA 96, 10585-10587.

Young, P. H. (1993): 'The Evolution of Conventions', Econometrica 61, 57-84. 